\title{
Variações morfológicas intra e interpopulacionais de Euseius citrifolius Denmark \& Muma e Euseius concordis (Chant) (Acari, Phytoseiidae)
}

\author{
Aloyséia Cristina da Silva Noronha ${ }^{1}$ \\ Gilberto José de Moraes ${ }^{2}$
}

\begin{abstract}
Intra and interpopulational morphological variations of Euseius citrifolius Denmark \& Muma and Euseius concordis (Chant) (Acari, Phytoseiidae). The determination of morphologic variability within and between populations of phytoseiid mites is important for the precise species identification. Euseius citrifolius Denmark \& Muma, 1970 and Euseius concordis (Chant, 1959) are phytoseiids commonly found on different crops in Brazil and other South American countries. The morphologic characterization of populations preliminarily identified as E. citrifolius and $E$. concordis was done through examination of 10 adult females and 10 adult males of each population and of 2 to 10 adult females and males resulting from crosses between those populations. The plant substrate and collection site of each population were: E. citrifolius: Bauhinia sp. in Arroio do Meio, Rio Grande do Sul, Coffea arabica Linnaeus in Campinas, São Paulo and Terminalia catappa Linnaeus in Petrolina, Pernambuco. E. concordis: Passiflora edulis Sims. f. flavicarpa Deg. in Arroio do Meio, Manihot esculenta (Crantz) in Jaguariúna, São Paulo, Hevea brasiliensis Muell. Arg. in Pontes e Lacerda, Mato Grosso, T. catappa in Petrolina and C. arabica in Viçosa, Minas Gerais. A comparison of the measurements of different structures of individuals of each population and of type specimens of E. citrifolius and E. concordis confirmed the preliminary identification of the populations. Significant relationships were observed between mean setal lengths and the respective ranges within each population. Females and males of $E$. citrifolius from Petrolina and $E$. concordis from Jaguariúna had some of the setae generally shorter than those of other populations of the same species. Measurements of males resulting from heterogamic crosses indicated that $E$. citrifolius and E. concordis reproduce by pseudo-arrhenotoky. KEY WORDS. Mite, phytoseiid, predator, pseudo-arrenotoky, taxonomy
\end{abstract}

Ácaros da família Phytoseiidae são eficientes inimigos naturais de ácaros pragas de vários cultivos. A identificação de ácaros dessa família é normalmente realizada pela avaliação de caracteres morfológicos. Entretanto, variações nos caracteres morfológicos entre indivíduos de uma população ou entre populações de uma espécie são sempre esperadas. O que comumente não se conhece, entretanto, é a amplitude esperada de variação de cada caráter considerado.

1) Embrapa Mandioca e Fruticultura. Caixa Postal 007, 43800-000 Cruz das Almas, Bahia, Brasil. E-mail: aloyseia @ cnpmf.embrapa.br

2) Departamento de Entomologia, Fitopatologia e Zoologia Agrícola, Escola Superior de Agricultura "Luiz de Queiroz", Universidade de São Paulo. Caixa Postal 9, 13418-900 Piracicaba, São Paulo, Brasil. Pesquisador CNPq. E-mail: gjmoraes@ carpa.ciagri.usp.br 
Poucos estudos no sentido de se conhecer as variações morfológicas de ácaros fitoseídeos têm sido conduzidos. Variaç̃̃es morfológicas entre oito populações de Galendromus longipilus (Nesbitt, 1951) e 12 populações de Galendromus occidentalis (Nesbitt, 1951) provenientes de diferentes regiões dos Estados Unidos da América foram observadas por HOYING \& CROFT (1977). Os autores verificaram que o comprimento do peritrema e da seta dorsal II foram os caracteres morfológicos que apresentaram diferença significativa, já que os demais caracteres não permitiram uma completa separação entre os dois grupos. MCMURTRY (1980) verificou que as variações morfológicas intra-específicas foram maiores entre 2 subespécies de Euseius addoensis (Van der Merwe \& Ryke, 1964) do que entre aquelas subespécies e uma população de Euseius citri (Van der Merwe \& Ryke, 1964).

Espécimes de Euseius mesembrinus (Dean, 1957) procedentes da Flórida e do Texas, EUA, apresentaram diferenças em relação ao comprimento de algumas setas. Esta variação foi atribuída a fatores geográficos ou nutricionais, já que foram verificadas apenas em indivíduos provenientes do campo, desaparecendo em condições de laboratório, onde as fontes de alimento e outros fatores ecológicos foram controlados (ABOU-SETTA et al. 1991).

No processo reprodutivo conhecido como pseudo-arrenotoquia, já demonstrado para algumas espécies de fitoseídeos, os machos são haplóides em decorrência da perda de um conjunto de cromossomos de origem paterna, apresentando basicamente a mesma constituição genética que suas mães (SCHULTEN 1985). PERROT-MinNOT \& NAVAJAS (1995) obtiveram evidências da contribuição genética paterna na produção de machos haplóides. Estudo recente conduzido por PERROT-MinNot et al. (2000) sugere que o material genético paternal presente nos descendentes machos da geração F1 não é transmitido para os descendentes de retrocruzamentos de fêmeas parentais e machos da geração F1. Entretanto, são necessários estudos para a comprovação de que as espécies de fitoseídeos utilizadas nesses trabalhos se reproduzam por pseudo-arrenotoquia. Assim sendo, evidências sobre o processo reprodutivo em espécies de fitoseídeos podem ser obtidas através de estudos de caracterização morfológica dos descendentes de cruzamentos envolvendo populações com algum nível de diferenças morfológicas. A indicação da ocorrência da pseudo-arrenotoquia em Euseius hibisci (Chant, 1959) e E. quetzali McMurtry, 1985 com base em estudos morfológicos foi relatada por CONGDON \& MCMURTRY (1988).

Exemplares das espécies utilizadas no presente estudo foram coletados na América do Sul. A espécie E. citrifolius Denmark \& Muma, 1970 foi descrita de espécimes coletados em Assunção, Paraguai, e E. concordis (Chant, 1959), de espécimes coletados em Concórdia, Entre Rios, Argentina. Ambas espécies estão entre os fitoseídeos mais comumente encontrados em diversas regiões do Brasil sobre plantas de folhas lisas (MORAES et al. 1986; FERLA \& MORAES 1998; FERES 2000). Além das descrições originais, informações complementares sobre a morfologia de E. citrifolius e E. concordis foram apresentadas por MORAES \& MCMURTRY (1983); MORAES et al. (1991); FERES \& MORAES (1998) e GONDIM JÚNIOR \& MORAES (2001). 
O presente trabalho teve por objetivo a determinação de variações morfológicas dentro e entre populações previamente identificadas como $E$. citrifolius e $E$. concordis, provenientes de diferentes plantas hospedeiras e regiões do Brasil, para confirmação das espécies, considerando que estudos de variações morfológicas entre e dentro de populações de predadores fitoseídeos mostraram consideráveis variações dentro de uma espécie de acordo com a distribuição geográfica, estação e planta hospedeira (MORAES 1987).

\section{MATERIAL E MÉTODOS}

Os substratos e locais de coleta de cada espécie estudada foram: E. citrifolius: Bauhinia sp. em Arroio do Meio, Rio Grande do Sul (R), Coffea arabica L. em Campinas, São Paulo (S) e Terminalia catappa L. em Petrolina, Pernambuco (P); E. concordis: Passiflora edulis Sims. f. flavicarpa Deg. em Arroio do Meio (R), Manihot esculenta (Crantz) em Jaguariúna, São Paulo (S), T. catappa em Petrolina, Pernambuco (P), Hevea brasiliensis Muell. Arg. em Pontes e Lacerda, Mato Grosso (M) e C. arabica em Viçosa, Minas Gerais (V). Os locais de coleta de E. citrifolius mais próximos entre si são Arroio do Meio e Campinas (aproximadamente $900 \mathrm{~km}$ em linha reta), e os locais mais distantes entre si são Arroio do Meio e Petrolina (aproximadamente $2.600 \mathrm{~km}$ ). Os locais de coleta de $E$. concordis mais próximos entre si são Jaguariúna e Viçosa (aproximadamente $500 \mathrm{~km}$ ), e os locais mais distantes entre si são Arroio do Meio e Petrolina.

A descrição morfológica de populações identificadas preliminarmente como E. citrifolius e E. concordis foi realizada através de medições de estruturas consideradas importantes para a separação de espécies neste gênero.

A avaliação das fêmeas constou de medições do comprimento e largura do escudo dorsal; comprimento, larguras anterior e posterior do escudo ventrianal; comprimento de macrosetas das pernas; distâncias entre as bases das setas esternais (ST1-ST3, ST2-ST2, ST5-ST5); comprimento das setas dorsais j1, j3, j4, j5, j6, J2, J5, z2, z4, Z1, Z4, Z5, s4, S2, S4, S5, r3, R1; comprimento do cálice da espermateca e comprimento dos dígitos fixo e móvel da quelícera (nomenclatura de ROWELL et al. 1978 e CHANT \& YOSHIDA-SHAUL 1991). A avaliação dos machos constou das mesmas medições efetuadas para as fêmeas, com exceção do comprimento do cálice da espermateca, da largura posterior do escudo ventrianal, das distâncias entre as bases das setas esternais, e do comprimento dos dígitos fixo e móvel da quelícera; foi medido neste caso o comprimento do espermadáctilo.

Foram medidos 10 fêmeas e 10 machos adultos provenientes de colônias de manutenção de cada população estudada, cada uma iniciada com 50 a 70 indivíduos. Foram ainda medidos de 9 a 10 fêmeas e 2 a 10 machos adultos resultantes dos cruzamentos (fêmea x macho) PR, RP, PS e SP de E. citrifolius, e fêmeas e machos adultos resultantes dos cruzamentos RS, SR, MS, SM, MR, RM, VS, SV, RP1, VP, $\mathrm{SP}_{1}$ e MP de E. concordis.

Os dados morfológicos foram comparados entre as populações pelos valores médios e amplitudes, sem o uso de métodos estatísticos. Foi realizada para cada população a análise de correlação entre os valores médios e as amplitudes de variação das medições das setas. 


\section{RESULTADOS}

\section{Euseius citrifolius Denmark \& Muma, 1970}

Nas fêmeas de cada população, as setas j1, j3, z4, Z5, s4, S4 e S5, e as macrosetas do genu e do tarso da perna IV apresentaram maiores amplitudes de variação (Tab. I). Verificou-se uma relação significativa $(\mathrm{p}<0,05)$ entre a dimensão média das setas e os valores das amplitudes de variação em cada população. Os coeficientes de correlação para as populações $\mathrm{R}, \mathrm{S}$ e $\mathrm{P}$ foram $0,81,0,83$ e 0,83 , respectivamente.

Pequenas diferenças foram encontradas entre fêmeas das diferentes populações em relação às medições de algumas estruturas. Entretanto, as setas j1, j3, S2 e S5 foram em média mais curtas nas fêmeas de Petrolina. Ao mesmo tempo, aquelas fêmeas eram menores, conforme indicado pelas menores dimensões do escudo dorsal. Alem disso, observou-se uma sobreposição parcial das amplitudes das dimensões do escudo dorsal e das setas citadas das diferentes populações.

As medições verificadas para as fêmeas de todas as populações estão de acordo com os dados apresentados por MORAES \& MCMURTRY (1983) para espécimes de $E$. citrifolius do Nordeste do Brasil, embora as setas j1 e S2 apresentem medições superiores às medições de fêmeas de Petrolina. As medições das estruturas das populações estudadas também estão de acordo com as medições de um parátipo depositado na Escola Superior de Agricultura "Luiz de Queiroz", coletado em Cecílio Baez, Paraguai, sobre Psidium guajava L., em 6 de janeiro de 1969 (Tab. I).

Nas fêmeas descendentes dos cruzamentos heterogâmicos realizados (PR, RP, PS e SP), verificou-se uma tendência em serem as setas j1, j3, S2 e S5 semelhantes às médias da população de Petrolina. As setas $\mathrm{j} 3, \mathrm{Z} 5$ e a macroseta do genu IV foram ligeiramente menores nos descendentes PR em relação ás demais populações. As fêmeas cuja população materna era de Petrolina apresentavam escudos dorsal e ventrianal ligeiramente menores, semelhantemente ao que se observou para as fêmeas da população materna. Por outro lado, praticamente em todos os descendentes de cruzamentos heterogâmicos, as amplitudes de variação de cada estrutura medida se sobrepuseram às de cada população, maiores amplitudes de foram observadas em relação às dimensões das setas z2, z4, Z5 e s4, e das macrosetas do genu e do tarso da perna IV. Verificou-se uma relação significativa $(\mathrm{p}<0,05)$ entre a dimensão média das setas e os valores das amplitudes de variação em cada população. Os coeficientes de correlação para as populações R, S e P foram respectivamente, $0,60,0,63$ e 0,90 . Na população $R$, a seta $\mathrm{z} 4$ apresentou amplitude de variação desproporcionalmente elevada.

Da mesma forma que para as fêmeas, foram encontradas pequenas diferenças entre machos das diferentes populações em relação às medições de algumas estruturas. Nos machos de Petrolina, as setas j3, z2, z4, Z5, s4, S2, S5 e as macrosetas da tíbia III e do genu IV foram em média mais curtas que nos machos de outros locais (Tab. II). Entretanto, aqueles eram de tamanho um pouco menores que os machos dos outros locais. Além disso, observou-se entretanto uma sobreposição parcial das amplitudes das dimensões do escudo dorsal e das setas citadas entre as diferentes populações. 
Tabela I. Dimensões (mínimo-máximo) $(\mu \mathrm{m})$ de fêmeas de populações identificadas como Euseius citrifolius procedentes de Arroio do Meio, Rio Grande do Sul (R), Campinas, São Paulo (S) e Petrolina, Pernambuco (P); $n=10$ para cada população.

\begin{tabular}{|c|c|c|c|c|}
\hline Parâmetros morfológicos (1) & Parátipo (2) & $\mathrm{R}$ & $\mathrm{s}$ & $\mathrm{P}$ \\
\hline CED & 312 & $326(317-338)$ & $321(313-327)$ & $304(286-318)$ \\
\hline LED & 230 & $233(221-246)$ & $230(224-240)$ & $220(213-230)$ \\
\hline \multicolumn{5}{|l|}{ Setas dorsais } \\
\hline j1 & 22 & $25(24-27)$ & $27(26-27)$ & $23(20-24)$ \\
\hline j3 & 23 & $25(22-27)$ & $23(22-24)$ & $21(16-24)$ \\
\hline $\mathrm{j} 4$ & 13 & $12(11-14)$ & $11(11-12)$ & $13(11-14)$ \\
\hline j5 & 13 & $12(11-14)$ & $12(11-14)$ & $12(11-12)$ \\
\hline j6 & 13 & $13(11-14)$ & $13(11-14)$ & $14(12-14)$ \\
\hline $\mathrm{J} 2$ & 14 & $14(14-14)$ & $14(14-15)$ & $15(14-16)$ \\
\hline J5 & 5 & $5(5-7)$ & $5(5-5)$ & $5(4-5)$ \\
\hline $\mathrm{z} 2$ & 18 & $18(16-20)$ & $18(16-19)$ & $16(16-16)$ \\
\hline z4 & 20 & $19(16-22)$ & $17(16-19)$ & $19(16-23)$ \\
\hline$z 5$ & 13 & $11(11-12)$ & $12(11-14)$ & $11(11-12)$ \\
\hline $\mathrm{Z1}$ & 13 & $14(12-14)$ & $14(12-14)$ & $14(12-14)$ \\
\hline$Z 4$ & 15 & $16(14-16)$ & $15(14-16)$ & $16(14-16)$ \\
\hline $\mathbf{Z} 5$ & 60 & $66(59-70)$ & $66(62-70)$ & $61(57-68)$ \\
\hline s4 & 31 & $33(30-35)$ & $30(27-32)$ & $31(27-35)$ \\
\hline S2 & 18 & $18(16-19)$ & $18(16-19)$ & $16(14-16)$ \\
\hline $\mathrm{S} 4$ & 18 & $20(16-22)$ & $19(16-19)$ & $18(16-20)$ \\
\hline S5 & 23 & $26(19-30)$ & $25(22-27)$ & $22(20-23)$ \\
\hline r3 & 18 & $18(16-19)$ & $18(16-19)$ & $16(14-18)$ \\
\hline $\mathrm{R} 1$ & 13 & $13(11-14)$ & $13(11-14)$ & $13(11-14)$ \\
\hline \multicolumn{5}{|l|}{ Macrosetas } \\
\hline Genu I & 18 & $16(14-19)$ & $15(14-16)$ & $15(11-18)$ \\
\hline Genu II & 19 & $20(19-22)$ & $20(19-22)$ & $20(19-22)$ \\
\hline Genu III & 23 & $25(19-27)$ & $26(24-28)$ & $23(22-24)$ \\
\hline Tíbia III & 20 & $21(19-22)$ & $22(20-24)$ & $19(18-22)$ \\
\hline Genu IV & 38 & $40(32-43)$ & $41(38-46)$ & $37(31-43)$ \\
\hline Tíbia IV & 28 & $28(27-30)$ & $30(27-32)$ & $28(27-30)$ \\
\hline Tarso IV & 51 & $56(49-62)$ & $55(53-59)$ & $53(43-59)$ \\
\hline ST1 - ST3 & 55 & $55(51-57)$ & $52(51-54)$ & $52(49-54)$ \\
\hline $\mathrm{ST} 2$ - ST2 & 65 & $64(62-65)$ & $66(65-68)$ & $63(59-68)$ \\
\hline ST5 - ST5 & 72 & $70(68-73)$ & $70(68-73)$ & $67(65-70)$ \\
\hline EVA-ant. & 48 & $54(49-57)$ & $50(46-54)$ & $48(43-51)$ \\
\hline EVA-post. & 72 & $75(73-76)$ & $75(70-76)$ & $67(62-73)$ \\
\hline EVA-comp. & 100 & $102(97-105)$ & $103(97-105)$ & $96(89-103)$ \\
\hline Cálice & 18 & $21(18-24)$ & $21(19-22)$ & $22(20-24)$ \\
\hline Digito fixo & 22 & $24(22-27)$ & $24(22-24)$ & $24(22-24)$ \\
\hline Digito móvel & 22 & $23(22-24)$ & $22(22-23)$ & $23(22-24)$ \\
\hline
\end{tabular}

1) (CED) Comprimento do escudo dorsal, (LED) largura do escudo dorsal, (ST1-ST3) distância entre as setas 1 e 3 do escudo esternal, (ST2-ST2) distância entre as setas ST2 do escudo esternal; (ST5-ST5) distância entre as setas do escudo genital, (EVA-ant.) largura da parte anterior do escudo ventrianal, (EVA-post.) largura da parte posterior do escudo ventrianal, (EVA-comp.) comprimento do escudo ventrianal; 2) Cecilio Baez, Paraguai, 06.I.1969, em Psidium guajava (Depositado na ESALQ/USP).

Nos machos PR e PS, as setas j3, z2, z4, Z5, s4, S2, S4, S5, e as macrosetas da tíbia III e do genu IV foram em média notoriamente mais curtas em relação às populações paternas R e S. Além disso, nos machos PR, as macrosetas do genu III, da tíbia IV e tarso IV, foram expressivamente mais curtas em relação à população paterna (Tab. II). 
As setas z2, z4, Z5, S2, S4, S5 e a macroseta do genu IV nos machos RP foram em média mais longas que nos machos da população paterna, embora tenha se observado a sobreposição das amplitudes de variação correspondentes (Tab. II).

Nos machos SP, as setas j3, S4, S5 e a macroseta do genu IV foram em média mais longas que nos machos da população paterna, embora os extremos inferiores das amplitudes de variação dessas estruturas nos machos SP estivessem próximos aos extremos superiores das amplitude correspondentes dos machos da população paterna (Tab. II). Nos machos PR e PS, as setas j1, j3, Z5, s4, S4, S5 e as macrosetas da tíbia III e genu IV foram em média mais curtas que nos machos RP e SP. Entretanto, também neste caso os extremos superiores das amplitudes de variação destas estruturas para os machos PR e PS estiveram sempre próximos aos extremos inferiores correspondentes dos machos RP e SP.

Tabela II. Dimensões (mínimo-máximo) $(\mu \mathrm{m})$ de machos de populações identificadas como Euseius citrifolius procedentes de Arroio do Meio, Rio Grande do Sul (R), Campinas, São Paulo (S), Petrolina, Pernambuco $(P)$ e de cruzamentos entre essas populações; $n=10$ para cada população e combinação.

\begin{tabular}{cccccccc}
\hline $\begin{array}{c}\text { Parâmetros } \\
\text { morfológicos (1) }\end{array}$ & $R$ & S & $P$ & $P R(2)$ & $R P(3)$ & PS (4) & SP (5) \\
\hline CED & $242(230-248)$ & $243(235-251)$ & $234(221-240)$ & $230(219-243)$ & $245(236-270)$ & $240(230-254)$ & $247(238-257)$ \\
LED & $194(186-202)$ & $183(176-189)$ & $173(159-184)$ & $183(167-189)$ & $190(176-200)$ & $190(173-200)$ & $190(181-197)$ \\
j1 & - & - & - & $18(16-19)$ & $21(19-22)$ & $17(14-19)$ & $20(19-22)$ \\
j3 & $24(22-27)$ & $25(22-27)$ & $20(19-22)$ & $20(19-22)$ & $22(19-27)$ & $19(16-22)$ & $23(22-24)$ \\
z2 & $19(16-22)$ & $19(16-22)$ & $15(14-16)$ & $16(14-16)$ & $18(16-19)$ & $15(14-16)$ & - \\
z4 & $19(16-27)$ & $19(16-22)$ & $16(15-18)$ & $16(14-19)$ & $18(16-19)$ & $16(16-16)$ & - \\
Z5 & $47(40-49)$ & $48(45-51)$ & $44(40-51)$ & $40(35-43)$ & $47(42-51)$ & $41(38-43)$ & $44(40-46)$ \\
S4 & $28(24-32)$ & $28(24-32)$ & $24(22-27)$ & $24(19-27)$ & $26(24-27)$ & $23(19-24)$ & $25(24-30)$ \\
S2 & $18(16-19)$ & $17(14-19)$ & $15(14-16)$ & $14(11-16)$ & $17(16-19)$ & $14(14-16)$ & - \\
S4 & $19(16-19)$ & $18(15-20)$ & $17(16-19)$ & $16(14-19)$ & $20(16-24)$ & $15(14-16)$ & $20(16-24)$ \\
S5 & $23(19-24)$ & $22(19-26)$ & $19(18-20)$ & $19(16-22)$ & $23(19-30)$ & $19(19-22)$ & $23(20-27)$ \\
Sge III & $20(19-22)$ & $19(18-19)$ & - & $15(14-16)$ & - & $17(16-19)$ & - \\
Sti III & $19(19-19)$ & $18(16-19)$ & $16(14-18)$ & $16(14-19)$ & $18(16-19)$ & $16(14-16)$ & $19(16-19)$ \\
Sge IV & $28(27-30)$ & $29(27-31)$ & $24(19-27)$ & $25(22-27)$ & $28(27-30)$ & $26(24-27)$ & $28(27-30)$ \\
Sti IV & $24(22-27)$ & $24(22-27)$ & - & $20(19-22)$ & - & $22(22-24)$ & - \\
St IV & $43(40-49)$ & $41(38-46)$ & - & $38(35-40)$ & - & $42(40-46)$ & - \\
\hline
\end{tabular}

1) (CED) Comprimento do escudo dorsal, (LED) largura do escudo dorsal, ( $j 1, j 3, z 2, z 4, z 5$, s4, S4, S5) setas localizadas no escudo dorsal, (Sge III) macroseta do genu da perna III, (Sti III) macroseta da tíbia da perna III, (Sge IV) macroseta do genu da perna IV, (Sti IV) macroseta da tíbia da perna IV, (St IV) macroseta do tarso da perna IV; 2) machos descendentes de fêmeas de Petrolina e machos de Arroio do Meio; 3) machos descendentes de fêmeas de Arroio do Meio e machos de Petrolina; 4) machos descendentes de fêmeas de Petrolina e machos de Campinas; 5) machos descendentes de fêmeas de Campinas e machos de Petrolina.

\section{Euseius concordis (Chant, 1959)}

Nas fêmeas de cada população, as maiores amplitudes de variação foram verificadas em relação às setas $\mathrm{j} 1, \mathrm{j} 3, \mathrm{z2}, \mathrm{z} 4, \mathrm{Z5}, \mathrm{s} 4 \mathrm{e}$ as macrosetas do genu das pernas III e IV, da tíbia das pernas III e IV e do tarso IV (Tab. III). Verificou-se uma relação significativa $(\mathrm{p}<0,05)$ entre as dimensões médias das setas e os valores das 
amplitudes de variação dentro de cada população. Maiores coeficientes de correlação foram obtidos nas populações de Petrolina $(0,80)$ e Jaguariúna $(0,73)$, para as demais populações este coeficiente variou de 0,47 a 0,62 .

Tabela III. Dimensões (mínimo-máximo) $(\mu \mathrm{m})$ de fêmeas de populações identificadas como Euseius concordis procedentes de Arroio do Meio, Rio Grande do Sul (R), Jaguariúna, São Paulo (S), Petrolina, Pernambuco (P), Pontes e Lacerda, Mato Grosso (M) e Viçosa, Minas Gerais $(V)$. ( $n=10$ para cada população).

\begin{tabular}{|c|c|c|c|c|c|c|}
\hline Parâmetros morfológicos (1) & Holótipo (2) & $\mathrm{R}$ & S & $\mathrm{P}$ & M & V \\
\hline CED & 317 & $326(313-340)$ & $315(302-332)$ & $307(292-338)$ & $315(292-325)$ & $312(300-321)$ \\
\hline LED & 230 & $233(219-248)$ & $232(224-246)$ & $224(204-246)$ & $227(213-236)$ & $222(211-230)$ \\
\hline \multicolumn{7}{|l|}{ Setas dorsais } \\
\hline$j 1$ & 30 & $28(27-30)$ & $24(22-27)$ & $26(24-32)$ & $27(24-30)$ & $27(27-28)$ \\
\hline j3 & 37 & $36(32-38)$ & $29(27-32)$ & $38(35-43)$ & $37(32-40)$ & $37(35-41)$ \\
\hline $\mathrm{j} 4$ & 10 & $8(8-8)$ & $8(7-8)$ & $9(8-1)$ & $8(7-8)$ & $8(8-11)$ \\
\hline j5 & 9 & $8(8-8)$ & $8(7-8)$ & $9(8-11)$ & $8(8-9)$ & $8(7-11)$ \\
\hline j6 & 13 & $11(8-11)$ & $9(8-11)$ & $11(9-14)$ & $9(8-11)$ & $9(8-11)$ \\
\hline $\mathrm{J} 2$ & quebrada & $11(11-11)$ & $10(8-11)$ & $11(9-14)$ & $10(9-11)$ & $9(8-11)$ \\
\hline J5 & quebrada & $5(4-5)$ & $5(4-5)$ & $5(5-5)$ & $5(4-5)$ & $5(5-5)$ \\
\hline$z 2$ & 17 & $20(16-24)$ & $16(14-19)$ & $23(19-28)$ & $18(14-22)$ & $20(19-22)$ \\
\hline $\mathrm{z} 4$ & 29 & $36(31-38)$ & $28(22-32)$ & $40(35-49)$ & $33(30-38)$ & $34(30-38)$ \\
\hline 25 & 9 & $8(8-8)$ & $8(7-8)$ & $8(7-9)$ & $8(7-9)$ & $8(8-8)$ \\
\hline $\mathrm{Z1}$ & 11 & $11(9-11)$ & $9(8-11)$ & $11(11-11)$ & $10(8-11)$ & $10(8-11)$ \\
\hline$Z 4$ & 11 & $11(11-11)$ & $11(11-11)$ & $11(11-14)$ & $11(8-11)$ & $11(8-11)$ \\
\hline $\mathrm{Z} 5$ & 64 & $63(59-65)$ & $56(43-62)$ & $61(57-65)$ & $63(59-65)$ & $61(58-62)$ \\
\hline s4 & 52 & $52(49-54)$ & $43(38-49)$ & $55(51-62)$ & $51(49-54)$ & $50(49-54)$ \\
\hline S2 & 14 & $15(14-16)$ & $14(11-16)$ & $14(12-16)$ & $14(11-14)$ & $14(11-16)$ \\
\hline S4 & 16 & $17(16-19)$ & $15(14-16)$ & $16(14-19)$ & $16(14-19)$ & $17(14-19)$ \\
\hline S5 & 18 & $20(19-22)$ & $17(16-19)$ & $20(18-23)$ & $17(14-19)$ & $17(14-19)$ \\
\hline r3 & 16 & $17(16-19)$ & $16(14-19)$ & $17(15-19)$ & $16(14-16)$ & $18(16-19)$ \\
\hline R1 & - & $11(11-11)$ & $10(8-11)$ & $10(9-11)$ & $11(11-11)$ & $12(11-14)$ \\
\hline \multicolumn{7}{|l|}{ Macrosetas } \\
\hline Genu I & - & $24(22-26)$ & $23(22-24)$ & $24(23-27)$ & $24(22-27)$ & $23(21-24)$ \\
\hline Genu II & 24 & $25(24-27)$ & $24(22-24)$ & $25(24-30)$ & $25(24-27)$ & $24(24-27)$ \\
\hline Genu III & 29 & $26(24-27)$ & $27(26-27)$ & $27(24-34)$ & $28(27-30)$ & $28(27-30)$ \\
\hline Tíbia III & - & $21(19-22)$ & $22(22-26)$ & $22(19-27)$ & $23(22-24)$ & $20(19-22)$ \\
\hline Genu IV & 41 & $40(38-43)$ & $37(35-38)$ & $39(34-49)$ & $40(39-43)$ & $41(38-41)$ \\
\hline Tíbia IV & 27 & $28(27-30)$ & $26(24-27)$ & $28(27-35)$ & $30(27-32)$ & $26(24-27)$ \\
\hline Tarso IV & 48 & $49(49-50)$ & $47(46-51)$ & $50(42-57)$ & $48(46-51)$ & $46(43-49)$ \\
\hline ST1-ST3 & - & $55(54-57)$ & $54(51-54)$ & $54(51-54)$ & $54(51-54)$ & $55(54-58)$ \\
\hline ST2-ST2 & - & $67(65-70)$ & $63(62-65)$ & $65(60-76)$ & $63(62-65)$ & $67(65-68)$ \\
\hline ST5-ST5 & - & $69(68-70)$ & $67(59-70)$ & $71(65-78)$ & $68(65-70)$ & $68(65-70)$ \\
\hline EVA-ant. & - & $51(49-54)$ & $47(43-51)$ & $50(46-54)$ & $51(49-54)$ & $49(46-51)$ \\
\hline EVA-post. & - & $71(69-76)$ & $69(65-76)$ & $68(65-73)$ & $70(68-73)$ & $69(68-70)$ \\
\hline EVA-comp. & - & $107(100-113)$ & $108(105-113)$ & $101(93-119)$ & $102(94-105)$ & $97(95-100)$ \\
\hline Cálice & - & $26(24-28)$ & $27(24-30)$ & $25(22-32)$ & $31(27-38)$ & $24(19-27)$ \\
\hline Dígito fixo & - & $24(24-24)$ & $23(20-24)$ & $24(22-24)$ & $24(22-25)$ & $23(22-24)$ \\
\hline Digito móvel & - & $24(22-24)$ & $22(20-24)$ & $23(22-24)$ & $23(22-24)$ & $22(19-24)$ \\
\hline
\end{tabular}

1) (CED) Comprimento do escudo dorsal, (LED) largura do escudo dorsal, (ST1-ST3) distância entre as setas 1 e 3 do escudo esternal, (ST2-ST2) distância entre as setas ST2 do escudo esternal, (ST5-ST5) distância entre as setas do escudo genital, (EVA-ant.) largura da parte anterior do escudo ventrianal, (EVA-post.) largura da parte posterior do escudo ventrianal, (EVA-comp.) comprimento do escudo ventrianal; 2) MCMURTRY (1983). 
As setas j3, z2, z4, s4 e a macroseta do genu IV das fêmeas de Jaguariúna foram em média mais curtas que nas fêmeas das outras populações, embora as fêmeas das populações S, M e V fossem aproximadamente do mesmo tamanho, como indicado pelas dimensões do escudo dorsal (Tab. III). As maiores diferenças foram observadas para as setas z2 e z4, que em alguns ácaros de Petrolina foram até 2 ou mais vezes maiores que em alguns ácaros de Jaguariúna. Por outro lado, os extremos superiores das amplitudes de variação destas estruturas para a população de Jaguariúna estiveram sempre próximos aos extremos inferiores correspondentes das outras populações. Não houve entretanto sobreposição entre as amplitudes de variação das setas j3, z4 e s4 entre as populações de Jaguariúna e Petrolina.

As medições verificadas para as fêmeas de todas as populações considerandose os valores das amplitudes de variação, estão de acordo com os dados apresentados por MORAES \& MCMURTRY (1983) para exemplares coletados no nordeste do Brasil, e em relação às medições do holótipo (MCMURTRY 1983) (Tab. III).

Em geral, as dimensões das estruturas consideradas foram muito semelhantes entre as fêmeas descendentes dos cruzamentos RS, MR, RM e VS, e as respectivas populações parentais. Entretanto, as dimensões das setas j3, z2, z4 e s4 apresentaram médias sempre superiores nas populações parentais $\mathrm{Me} \mathrm{R}$, em relação às dimensões das estruturas correspondentes nos descendentes MR e RM, embora tenha se observado a sobreposição das amplitudes de variação correspondentes (Tab. IV). Ainda com relação às mesmas setas, as fêmeas RS e VS apresentaram dimensões médias inferiores àquelas das fêmeas das populações maternais correspondentes, e superiores àquelas das fêmeas da população paternal, embora as amplitudes de variação também se sobrepusessem.

Nos machos de cada população, maiores amplitudes de variação foram observadas em relação às setas $\mathrm{j} 1, \mathrm{z} 4, \mathrm{Z5}, \mathrm{s} 4, \mathrm{~S} 5$ e macrosetas do genu da perna IV e do tarso IV (Tab. V). Verificou-se uma relação significativa $(\mathrm{p}<0,05)$ entre as dimensões média das setas e os valores das amplitudes de variação para cada população. Maiores coeficientes de correlação foram obtidos nas populações de Jaguariúna $(0,76)$ e Arroio do Meio $(0,70)$; nas demais populações este coeficiente variou de 0,54 a 0,64 .

A maior parte das estruturas não apresentou diferenças marcantes entre as distintas populações. Na população de Jaguariúna, apenas a seta $\mathrm{z} 4$ apresentou-se marcadamente curta, mas o extremo superior de sua amplitude de variação coincidiu com o extremo inferior das amplitudes correspondentes das populações de Petrolina e Arroio do Meio e foi ainda maior que o extremo inferior das amplitudes correspondentes das populações de Pontes e Lacerda e Viçosa (Tab. V).

Nos machos RS, as setas z4, s4, S4 e S5 apresentaram dimensões médias superiores às médias da população paternal, mas as amplitudes de variação correspondentes, para machos RS e machos S se sobrepuseram (Tab. V). Nos machos SR, a seta $s 4$ foi em média mais curta que a mesma nos machos da população paterna, mas o extremo superior de amplitude de variação de SR coincidiu com o limite inferior da amplitude correspondente da população paterna (Tab. V). 
Tabela IV. Dimensões (mínimo-máximo) $(\mu \mathrm{m})$ de fêmeas de populações identificadas como Euseius concordis e descendentes de cruzamentos entre populações procedentes de Arroio do Meio, Rio Grande do Sul (R), Jaguariúna, São Paulo (S), Petrolina, Pernambuco (P), Pontes e Lacerda, Mato Grosso (M) e Viçosa, Minas Gerais (V); $n=10$ para cada população e combinação.

\begin{tabular}{|c|c|c|c|c|c|c|c|c|c|}
\hline Parâmetros morfológicos (1) & $\mathrm{R}$ & $\mathrm{S}$ & $\mathrm{P}$ & M & v & MR (2) & $\mathrm{RM}(3)$ & $\operatorname{RS}(4)(n=9)$ & VS 5 \\
\hline j3 & $36(32-38)$ & $29(27-32)$ & $38(35-43)$ & $37(32-40)$ & $37(35-41)$ & $32(27-35)$ & $31(30-32)$ & $30(30-35)$ & $33(28-35)$ \\
\hline$z 2$ & $20(16-24)$ & $16(14-19)$ & $23(19-28)$ & $18(14-22)$ & $20(19-22)$ & $16(14-19)$ & $16(14-19)$ & $18(16-22)$ & $18(16-19)$ \\
\hline$z 4$ & $36(31-38)$ & $28(22-32)$ & $40(35-49)$ & $33(30-38)$ & $34(30-38)$ & $28(24-30)$ & $27(24-28)$ & $30(27-32)$ & $30(27-35)$ \\
\hline
\end{tabular}

1) (j3, z2, z4, s4) setas do escudo dorsal, (Sge IV) macroseta do genu da perna IV; 2) fêmeas descendentes de fêmeas de Pontes e Lacerda e machos de Arroio do Meio; 3) fêmeas descendentes de fêmeas de Arroio do Meio e machos de Pontes e Lacerda; 4) fêmeas descendentes de Arroio do Meio e machos de Jaguariúna; 5) fêmeas descendentes de Viçosa e machos de Jaguariúna.

Tabela V. Dimensões (mínimo-máximo) $(\mu \mathrm{m})$ de machos de populações identificadas como Euseius concordis e de descendentes de cruzamentos entre essas populações procedentes de Arroio do Meio, Rio Grande do Sul (R), Jaguariúna, São Paulo (S), Petrolina, Pernambuco (P), Pontes e Lacerda, Mato Grosso (M) e Viçosa, Minas Gerais (V). ( $n=10$ para cada população e combinação).

\begin{tabular}{|c|c|c|c|c|c|c|c|c|c|c|c|c|}
\hline Parâmetros morfológicos (1) & $R$ & S & $\mathrm{P}$ & M & v & RS (2) & SR (3) & $\mathrm{SM}(4)$ & MR (5) & VS (6) & SV (7) & SP (8) \\
\hline CED & $248(239-255)$ & $42(235-248)$ & $233(230-238)$ & $35(216-247$ & $244(235-254)$ & - & - & - & - & - & - & - \\
\hline LED & $190(184-197)$ & $90(176-200)$ & $189(181-208)$ & $200(194-205$ & $187(173-205)$ & - & - & - & - & - & - & - \\
\hline $\mathrm{j} 3$ & - & - & - & $32(30-32)$ & - & - & - & $27(24-28)$ & - & - & - & - \\
\hline$z 2$ & - & - & $25(22-27)$ & - & - & $22(20-24)$ & - & - & - & - & $18(16-19)$ & $17(16-19)$ \\
\hline $\mathrm{z} 4$ & $31(30-36)$ & $27(22-30)$ & $32(30-36)$ & $31(27-32)$ & $31(27-32)$ & $31(30-32)$ & - & - & - & $27(26-30)$ & $32(30-40)$ & $27(24-27)$ \\
\hline Z5 & $50(46-54)$ & - & - & - & - & - & - & - & - & $45(38-49)$ & - & - \\
\hline s4 & $40(39-43)$ & $35(32-40)$ & - & - & - & $40(38-43)$ & $35(30-38)$ & - & - & $39(38-40)$ & - & - \\
\hline S4 & & & - & - & - & & - & - & - & & - & - \\
\hline S5 & $20(19-22)$ & $18(14-22)$ & - & - & - & $21(19-22)$ & - & - & - & $16(14-18)$ & - & - \\
\hline
\end{tabular}

1) (CED) Comprimento do escudo dorsal, (LED) largura do escudo dorsal, (j3, z2, z4, Z5, s4, S4, S5) setas do escudo dorsal; (2) machos descendentes de fêmeas de Arroio do Meio e machos de Jaguariúna; 3) machos descendentes de fêmeas de Jaguariúna e machos de Arroio do Meio; 4) machos descendentes de fêmeas de Jaguariúna e machos de Pontes e Lacerda; 5) machos descendentes de fêmeas de Pontes e Lacerda e machos de Arroio do Meio; 6) machos descendentes de fêmeas de Viçosa e machos de Jaguariúna; 7) machos descendentes de fêmeas de Jaguariúna e machos de Viçosa; 8) machos descendentes de fêmeas de Jaguariúna e machos de Petrolina. 
Os machos SM e MS, apresentaram as dimensões da maior parte das estruturas semelhantes àquelas dos machos das populações parentais, com exceção da seta j3, que foi em média mais curta nos machos SM que nos machos da população de Pontes e Lacerda, não havendo sobreposição das amplitudes de variação (Tab. IV). As dimensões da maior parte das estruturas os machos MR e RM foram semelhantes àquelas dos machos das populações parentais, com exceção das setas z4, Z5, S4 e S5, que foram em média mais curtas nos machos MR que nos machos da população paterna. As amplitudes das dimensões das setas S4 e S5 dos machos MR não se sobrepuseram às amplitudes correspondentes dos machos da população paterna (Tab. V).

As setas z4 e s4 foram em média mais longas nos machos VS que nos machos da população paterna, com as amplitudes de variação se sobrepondo. Machos SV apresentaram a seta z2 em média mais curta que nos machos da população paterna, com as amplitudes de variação não se sobrepondo (Tab. IV).

Nos machos RP1, VP e MP as dimensões das estruturas pouco variaram em relação às estruturas correspondentes nos machos das populações parentais.

As setas z2 e z4 apresentaram-se marcadamente mais curtas nos machos $\mathrm{SP}_{1}$ que nos machos da população paterna, sem haver sobreposição das amplitudes de variação (Tab. V).

\section{DISCUSSÃo}

A correlação significativa verificada para cada população de $E$. citrifolius sugere que as amplitudes de variação estão relacionadas com a comprimento das estruturas. Assim sendo, maiores amplitudes de variação devem ser esperadas para as setas e macrosetas de maior comprimento e vice-versa. Portanto, as setas j1, j3, z2, z4, Z5, s4, e as macrosetas do genu e do tarso IV devem apresentar maiores amplitudes de variação. Entretanto, a seta $\mathrm{Z} 4$ na população $\mathrm{R}$ fugiu à regra. Apesar desta seta apresentar menor dimensão que as setas j3, Z5 e s4, o valor da amplitude de variação foi marcadamente superior.

Apesar dos exemplares de E. citrifolius avaliados neste trabalho serem procedentes de três regiões geograficamente distantes, não foram constatadas diferenças expressivas nas dimensões das estruturas entre as populações. Consequentemente, também os indivíduos procedentes dos cruzamentos interpopulacionais apresentaram pequenas variações em relação às populações parentais. As maiores dimensões verificadas neste estudo para algumas setas do escudo dorsal de fêmeas em relação às dimensões das setas correspondentes no holótipo (DENMARK \& MUMA 1970) também foram observadas por MORAES \& MCMURTRY (1983), FERES \& MORAES (1998) e GONDIM JÚNIOR \& MORAES (2001), para exemplares de São Paulo e do Nordeste do Brasil. Entretanto, as medições neste estudo estão de acordo com as medições do parátipo.

WYSOKI \& MCMURTRY (1977) verificaram que E. citrifolius apresenta machos com quatro e fêmeas com oito cromossomos. Se considerarmos que os machos haplóides de $E$. citrifolius apresentam basicamente a mesma constituição genética que sua mãe, de acordo com o processo reprodutivo de pseudo-arrenoto-

Revta bras. Zool. 19 (4): 1111 - 1122, 2002 
quia, as semelhanças verificadas nas dimensões médias dos parâmetros estudados entre os machos PR, RP, PS e SP e os machos das populações maternais, assim como as diferenças observadas entre os machos híbridos e os machos das populações paternais, seriam esperadas.

Os resultados obtidos neste trabalho indicam que $E$. concordis também se reproduz pelo processo de pseudo-arrenotoquia, seguindo o padrão de machos haplóides e fêmeas diplóides. Nas fêmeas RS, MR, RM e VS, as dimensões médias dos parâmetros estudados não apresentaram variações expressivas quando comparadas com as dimensões de fêmeas de populações parentais; as setas j3, z2, z4 e s4 apresentaram pequenas diferenças, mas as amplitudes de variação se sobrepuseram em todas as comparações.

As semelhanças verificadas entre machos SR, RS, SR, VS, SV e SP1, e os machos das populações maternais também seriam esperadas, no processo de reprodução conhecido como pseudo-arrenotoquia.

Este processo reprodutivo também foi sugerido por CONGDON \& MCMURTRY (1988) para outras espécies de Euseius. Esses autores verificaram diferenças ecológicas e morfológicas entre $E$. hibisci e E. quetzali procedentes da Califórnia, entretanto produziram híbridos em condições de laboratório apresentando um isolamento parcial. Machos resultantes do cruzamento de fêmeas $E$. hibisci e machos $E$. quetzali foram semelhantes aos machos de $E$. hibisci, sugerindo que $E$. hibisci se reproduz por pseudo-arrenotoquia (parahaploidia).

A comparação dos dados morfológicos das populações estudadas neste trabalho com os dados de descrições disponíveis na literatura para E. citrifolius confirmam que as populações procedentes de Arroio do Meio, Campinas e Petrolina pertencem à esta espécie. Do mesmo modo, as populações procedentes de Arroio do Meio, Jaguariúna, Petrolina, Pontes e Lacerda e Viçosa pertencem à espécie $E$. concordis. As variações observadas nas medições de estruturas das populações de cada espécie, permitiram a diferenciação morfológica da população de E. citrifolius de Petrolina das populações de Arroio do Meio e de Campinas, assim como, a diferenciação morfológica da população de E. concordis de Jaguariúna, das demais populações correspondentes para esta espécie. A análise dos parâmetros morfológicos de machos de E. citrifolius e E. concordis provenientes de cruzamentos entre populações diferentes sugerem que estas espécies se reproduzem por pseudo-arrenotoquia.

\section{REFERÊNCIAS BIBLIOGRÁFICAS}

Abou-Setta, M.M.; C.C. Childers; H.A. Denmark \& H.W. Browning. 1991. Comparative morphology and reproductive compatibility between populations of Euseius mesembrinus (Acari: Phytoseiidae) from Florida and Texas. Exp. Appl. Acarol., Amsterdam, 10: 213-220.

Chant, D.A. \& E. Yoshida-Shaul. 1991. Adult ventral setal patterns in the family Phytoseiidae (Acari: Gamasida). Internat. Jour. Acarol., Michigan, 17 (3): 187.

Congdon, B.D. \& J.A. McMurtry. 1988. Morphological evidence establishing the loss of paternal chromosomes in males of predatory phytoseiid mites, genus Euseius. Entomol. Exp. Appl., Dordrecht, 48: 95-96.

Denmark, H.A. \& M.H. Muma. 1970. Some phytoseiid mites of Paraguay (Phytoseiidae: Acarina). Florida Entomol., Gainesville, 53 (4): 219-227. 
FERES, R.J.F. 2000. Levantamento e observações naturalísticas da acarofauna (Acari, Arachnida) de seringueiras cultivadas (Hevea spp., Euphorbiaceae) no Brasil. Revta bras. Zool., Curitiba, 17 (1): 157-173.

Feres, R.J.F. \& G.J. Moraes. 1998. Phytoseiid mites (Acari: Phytoseiidae) from woody areas in the State of São Paulo, Brazil. Syst. Appl. Acarol., Londres, 3: 125-132.

FerLa, N.J. \& G.J. Moraes. 1998. Ácaros predadores em pomares de maçã no Rio Grande do Sul. An. Soc. Entomol. Brasil, Londrina, 27 (4): 649-654.

Gondim Júnior, M.G.C. \& G.J. Moraes. 2001. Phytoseiid mites (Acari: Phytoseiidae) associated with palm trees (Arecaceae) in Brazil. Syst. Appl. Acarol., Londres, 6: 65-94.

Hoying, S.A. \& B.A. Croft. 1977. Comparisons between populations of Typhlodromus longipilus Nesbitt and T. occidentalis Nesbitt: Taxonomy, distribution, and hybridization. Ann. Entomol. Soc. Amer., College Park, 70 (1):150-159.

MCMURTRY, J.A. 1980. Biosystematics of three taxa in the Amblyseius finlandicus group from South Africa, with comparative life history studies (Acari: Phytoseiidae). Internat. Jour. Acarol., Michigan, 6 (2): 147-156.

-1983. Phytoseiid mites from Guatemala, with descriptions of two new species and redefinitions of the genera Euseius, Typhloseiopsis, and the Typhlodromus occidentalis species-group (Acari: Mesostigmata). Internat. Jour. Acarol., Michigan, 25 (4): 249-272.

MoraES, G.J. 1987. Importance of taxonomy in biological control. Insect Sci. Applic., Nairobi, 18 (4/6): 841-844.

Moraes, G.J. \& J.A. McMurtry. 1983. Phytoseiid mites (Acarina) of northeastern Brazil with descriptions of four new species. Internat. Jour. Acarol., Michigan, 9 (3):131-148.

Moraes, G.J.; J.A. McMurtry \& H.A. Denmark. 1986. A catalog of the mite family Phytoseiidae: references to taxonomy, synonymy, distribution and habitat. Brasília, EMBRAPA-DDT, 353p.

Moraes, G.J.; N.C. Mesa \& A. Braun. 1991. Some phytoseiid mites of Latin America (Acari: Phytoseiidae). Internat. Jour. Acarol., Michigan, 17 (2): 117-139.

Perrot-Minnot, M.J. \& M. NAVAJAs. 1995. Biparental inheritance of RAPD markers in males of the pseudo-arrhenotokous mite Typhlodromus pyri. Genome, Ottawa, 38: 838-844.

Perrot-Minnot, M.J.; J. LAgnel; A. Migeon \& M. Navajas. 2000. Tracking paternal genes with DALP markers in a pseudoarrhenotokous reproductive system: biparental transmission but haplodiploid-like inheritance in the mite Neoseiulus californicus. Heredity, Edinburgh, 84: 702-709.

Rowell, H.J.; D.A. Chant \& I.C. Hansell. 1978. The determination of setal homologies and setal patterns on the dorsal shield in the family Phytoseiidae (Acarina: Mesostigmata). Can. Entomol., Ottawa, 110: 859-876.

Schulten, G.G.M. 1985. Mating. p.55-65. In: W. Helle \& M.W. Sabelis (Eds). Spider mites their biology, natural enemies and control. Amsterdam, Elsevier, 203p.

WYSOKI, M. \& J.A. MCMURTRY. 1977. Karyotypes of eight species of phytoseiid mites of the genus Amblyseius Berlese (Acarina: Mesostigmata). Genetica, The Hague, 47 (3): 237-239.

Recebido em 11.I.2002; aceito em 11.X.2002. 